\title{
NEIGHBOUR ALPHA STABLE FUSION IN WAVELET DECOMPOSITION AND LAPLACIAN PYRAMID
}

\author{
Rachid Sabre ${ }^{1}$ and Ias Wahyuni ${ }^{2}$ \\ ${ }^{1}$ Laboratory Biogéosciences CNRS, University of \\ Burgundy/Agrosup Dijon, France \\ ${ }^{2}$ Universitas Gunadarma, J1. Margonda Raya No 100 Depok 16424, Indonesia
}

\begin{abstract}
In this paper, a new multifocus image fusion method is proposed, which combines the Laplacian pyramid, wavelet decomposition and uses alpha stable distance as a selection rule. First, using Laplacian pyramid, we decomposed the multifocus images into several levels of pyramid and then applied wavelet decomposition at each level. The contribution of this work is to fuse the wavelet images at each level by using alpha stable distance as a selection rule. To get the final fused image, we reconstructed the combined image at every level of the pyramid. This protocolwas compared to other methods and showed good results.
\end{abstract}

\section{KEYWORDS}

Image fusion, Laplacian pyramid, Wavelet decomposition

\section{INTRODUCTION}

Image fusion is the process of combining relevant information from two or more images into a single image that will be more informative than any input images. The goal of image fusion is to reduce uncertainty and minimize redundancy in the output as well as to maximize relevant information for an application or a task. With the rapid advancements in technology, it is now possible to obtain information from multiple source images to produce high quality information from a set of images. In this paper, we dealt with multi-focus images. Due to the limited depth-offocus of optical lenses in camera devices, it is often not possible to get an image thatcontains all relevant object 'in focus' so that one scene can be decomposedinto set of images with different focus of every image. We can use image fusion method to obtain all focused objects.

Many methods exist to perform image fusion [1]-[3]. In this work, we used Laplacian pyramid (LP) and the discrete wavelet transform (DWT) image fusion. The LP image fusion and DWT image fusion are multiscale transformation image fusion.

The LP image fusion integrates multi-source information at the basic level and can provide more abundant, accurate and reliable detailed information. The important thing in the LP image fusion is to define a selection rule for determining the value of each pixel in the resulting fused pyramid. The averaging method, maximum method, saliency and match measure [4], and combination of averaging and maximum energy method [4] have been used as selection rulesin LP image fusion. Recently, [6] used PCA as selection rule in LP image fusion and wavelet decomposition with maximum absolute selection rule [7]. 
The wavelet fusion method allows the image decomposition in different kind of coefficients subbands. Image fusion using wavelet method can be seen in [8]-[11]. The wavelet transformation modulus maxima gives better preservation of both edge features and component information of the object in new fused image preserving the detailed image information [7].

This work proposesa new multifocus image fusion method using (combination Laplacian Pyramid (LP) and wavelet transform fusion method with alpha stable distance as selection rule. Indeed, he usesdiscrete wavelet decomposition in each level of LP before undergoing fusion. The fusion rule is based on alpha distance of wavelet coefficients. This fusion method improved significantly the resulting fused image. This paper is organized as follows: Section 2 briefly gives explanation about neighbour alpha stable distance and Fusion. TheSection 3 developthe proposed method. The section 4 apply the proposed method on real images and compare it to other methods. The section 5 presents our conclusions and pinpoint potential perspectives.

\section{Neighbour Alpha Stable Distance AND Fusion}

The Alpha-stable distribution is widely used in the processing of impulsive or spiky signals. It has also been applied in image processing. [12] and [13] give the model of the sea clutter in SAR images using alpha stable distribution for ship detection while [14] removes speckle noise using alpha stable based Bayesian algorithm in the wavelet domain. Furthermore, alpha stable distribution is also used in image segmentation [15] and compressive image fusion [12]. Both [14], [15], and [13] and Wan employ alpha stable in wavelet domain. This section provides a brief of the alpha-stable distribution.

\section{A. Neighbor Alpha Stable Distance}

This workdevelop a novel fusion method as a selection rule in Laplacian pyramid method. This consisted of weighting each pixel of each image by exponential of Neighbor Alpha Stable Distance (NASD). This neighbor alpha stable distancegeneralize the quadratic difference between the value of the pixel $(x, y)$ and the all pixel values of its neighborswhen $\alpha=2$.The neighbor, with the size "a", of a pixel $(x, y)$ is defined as follows:

$(x+i, y+j)$ where $i=-a,-a+1, \ldots, a-1, a$ and $j=-a,-a+1, \ldots, a-1, a$.

For example the neighbour with the small size $\left(" a^{\prime \prime}=1\right)$ contains: $(x-1, y-1),(x-1, y)$, $(x-1, y+1),(x, y-1),(x, y+1),(x+1, y-1),(x+1, y),(x+1, y+1)$.

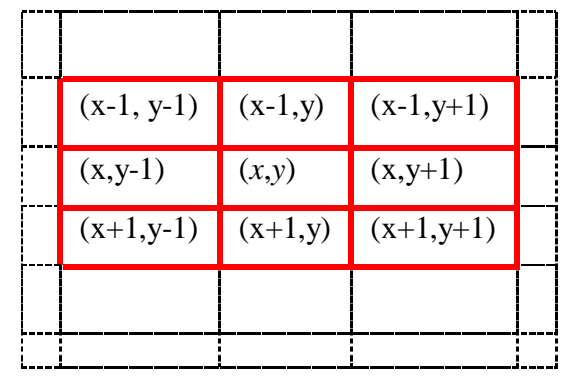

Figure 1. Pixel at $(x, y)$ within its neighborhood, $a=1$.

Then, the steps of image fusion with size " $a$ "wereas follows:

Let $I_{1}, \ldots, I_{M}$ be $M$ original source images with different focus to fuse. The images here had the same size $\left(N_{1} \times N_{2}\right)$. The general principle of making fusion rules were:

Step 1: For each pixel of each image, we calculated the neighbour alpha stable distance (NASD) of every source image, $d_{a, k}(x, y)$ : 
$d_{a, k}(x, y)=\left(\frac{1}{R} \sum_{m=-a}^{a} \sum_{n=-a}^{a}\left|I_{k}(x, y)-I_{k}^{\prime}(x+m, y+n)\right|^{\alpha}\right)^{\frac{1}{\alpha}}$

Where $I_{k}^{\prime}(x+m, y+n)=\left\{\begin{array}{l}I_{k}(x+m, y+n), \text { if } 1 \leq x+m \leq N_{1} \text { and } 1 \leq y+n \leq N_{2}, A=(2 a+1)^{2}-\operatorname{card}(S), \\ I_{k}(x, y), \text { otherwise }\end{array}\right.$ $S=\left\{(m, n) \in\left([-a, a]^{2}-\{(0,0)\}\right) \mid I_{k}^{\prime}(x+m, y+n)=I_{k}(x, y)\right\} . k=1, \ldots, M$.

\section{B. Neighbor Alpha Stable Fusion}

The Neighbour Alpha Stable Fusion (NASF) proposed in this work consisted of fused images using the following model:

$$
\operatorname{NASF}(x, y)=\frac{\sum_{i=1}^{M} \exp \left(d_{a, k}(x, y)\right) I_{i}(x, y)}{\sum_{i=1}^{M} \exp \left(d_{a, k}(x, y)\right)}
$$

Obviously, this method depended on the size "a". We have noticed that,for almost all images, a=4 gave the best result. In the following we took $a=4$.

\section{The Proposed Method}

The image fusion methods keep progressing to get the better result of fused image. In this work, we fused images using combination Laplacian pyramid, wavelet transform and NASF methods. First, we decomposed each source image by Laplacian pyramid (LP). This method was first introduced in [16] as a model for binocular fusion in human stereo vision, where the implementation used a Laplacian pyramid and a maximum selection rule at each point of the pyramid transformation [17]. Essentially, the procedure involved a set of band-pass copies of an image, referred to the Laplacian pyramid due to its similarity to a Laplacian operator. Each level of the Laplacian pyramid wasrecursively constructed from its lower level by applying the following four basic steps: blurring (low-pass filtering), sub-sampling (reduce size), interpolation (expand), and differencing (to subtract two images pixel by pixel). In the LP, the lowest level of the pyramid wasconstructed from the original image.

After the LP decomposition, we appliedDecomposition Wavelet Transform (DWT)at each level of Laplacian pyramid. The decomposition DWT used in this paper wasbased on Haar wavelet transform. DWT separately filtered and lowered samples images in the horizontal direction and vertical directions. This produced four coefficient subbands at each scale. As presented in [7], we supposed an image $I(x, y)$ and denoted the horizontal frequency first by using 1-D lowpass filter $\mathrm{L}$ and highpass filter $\mathrm{H}$ produces the coefficient matrices $I_{L}(x, y)$ and $I_{H}(x, y)$ and then followed the vertical frequency second by using lowpass filter $\mathrm{L}$ and highpass filter $\mathrm{H}$ to each column in $I_{L}(x, y)$ and $I_{H}(x, y)$. Thisproduced four sub-images $I_{L L}(x, y), I_{L H}(x, y), I_{H L}(x, y)$ , and $I_{H H}(x, y)$ for one level of decomposition. By recursively applying the same scheme to the low-low subband amultiresolution decomposition could be achieved.

After decomposing DWT, we used the NASF fusion defined in (6) to fuse images in wavelet decomposition by merging the DWT coefficient of every corresponding frequency band.

The fusion follows 4 steps: we supposed there were two original source images, A and B, with different focus:

1) Performing Laplacian pyramid decomposition to create Laplacian pyramid for each source image, 
2) Performing discrete wavelet decomposition to every level of Laplacian pyramid for each image in different kinds of coefficient,

3) Merging an appropriate coefficient of the corresponding subband to obtain new coefficients by using Neighbor Alpha Stable Fusion (NASF). The fused wavelet image is achieved through the inverse discrete wavelet transform,

4) The final fused image wasobtained by performing pyramid inverse transform on the fused wavelet image by using Neighbor Alpha Stable Fusion (NASF).

The process of pyramid image fusion can be seen in Fig.2, in which the fusion LPi's (i=1, 2, 3) wasobtained by applying wavelet transform and (NASF) fusion. Actually, it couldbe extended to more than twosource images A and B.

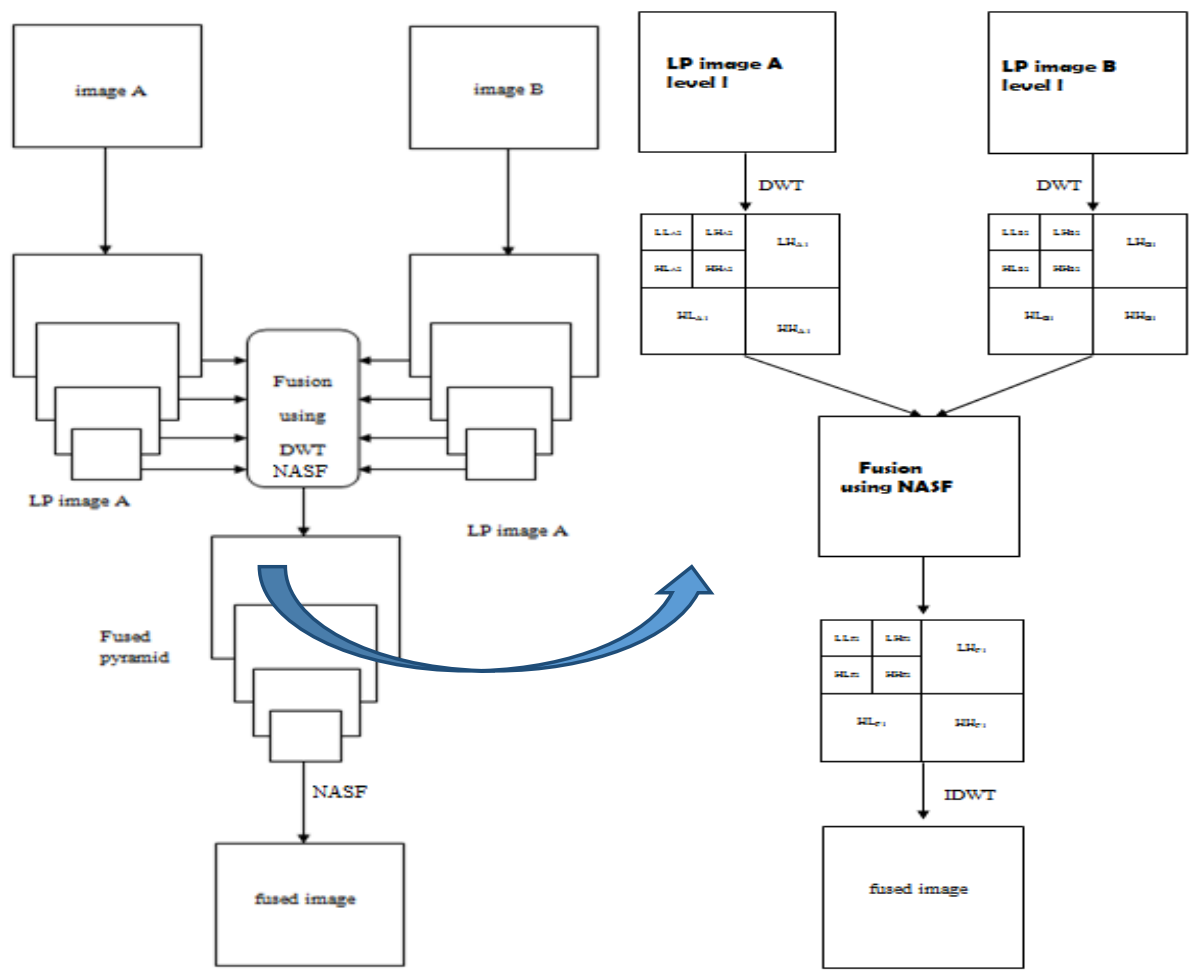

Figure 2. The proposed method Figure 3. DWT image fusion

The figure 3 shows the process of decomposition wavelet transform of LP images at level $l$ and fusion image using NASF method.

\section{EXPERIMENTAL RESULT}

As in the work [18], the images used in this section weretaken from the database [12]. We have blurred an area of each imageusing the convolution of Gaussian filter applied on the reference image $g_{f}$ :The choice of Gaussian is approved in the works [19]-[20].Blurred areas are chosen to hide an object from the photographedscene when there aremultiple objects. Thus, the size of blurred areas varied according tothe size of the objects hidden in the images. Let $g_{f}$ thereference image. The blurred image $g_{0}$ wasdefined as follows:

$g_{0}(i, j)=\left\{\begin{array}{l}\sum_{n=-2}^{2} \sum_{n=-2}^{2} h\left(m^{\prime}, n^{\prime}\right) g_{f}\left(i-m^{\prime}, j-n^{\prime}\right),(i, j) \in \text { blurred area } \\ g_{f}(i, j),(i, j) \in \text { object focus area }\end{array}\right.$ 
where $h\left(m^{\prime}, n^{\prime}\right)$ is Gaussian filter. We applied the method on 150 sets of multi focus images on a datasets of images [12]. In this paper, we presented only five set of multi focus images. Figures4, $6,8,10$ and 12 show the multi focus images obtained by the convolution of Gaussian filter. Figures 5,7, 9, 11 and 13 show the fused image by proposed method. Visually the image obtained by the proposed method gives a very satisfactory fusion.

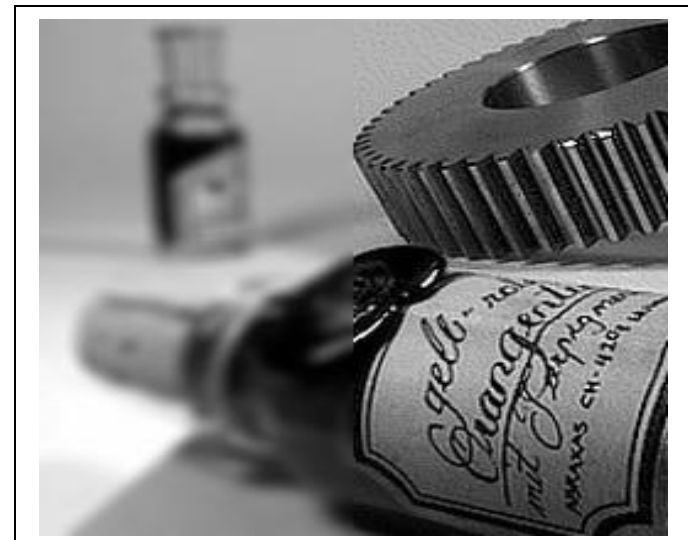

Blurred image1

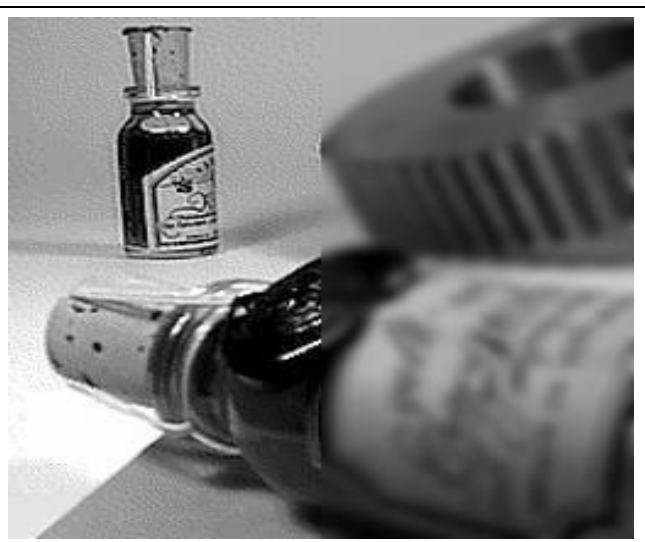

Blurred image2

Figure4. Multi focus images (bottles)

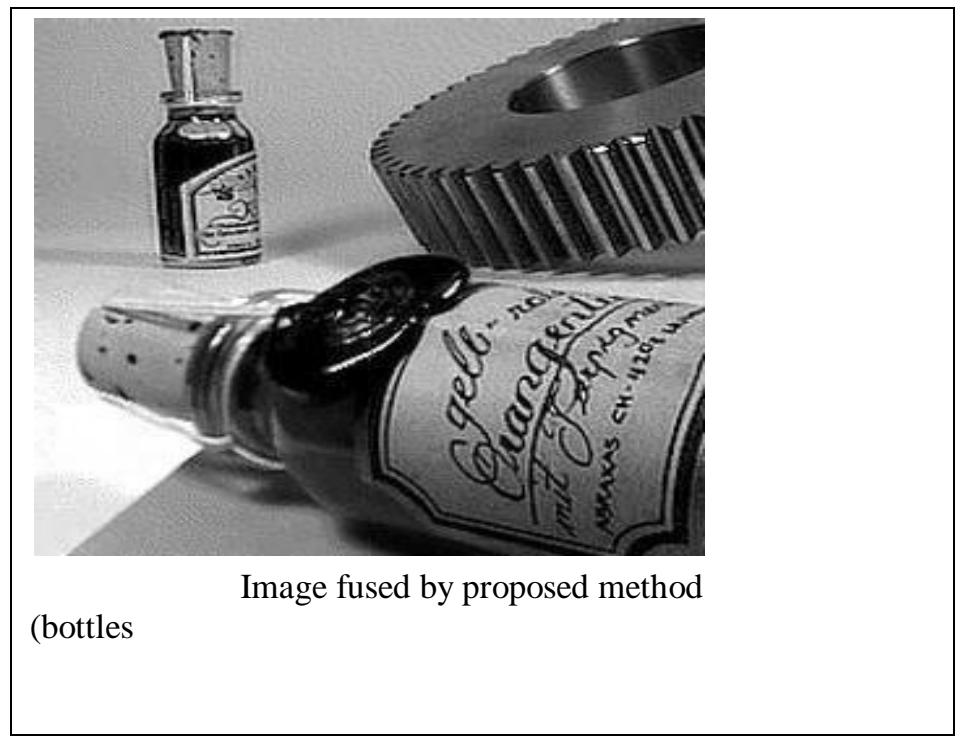

Figure5. Fused image by proposed method (bottles) 


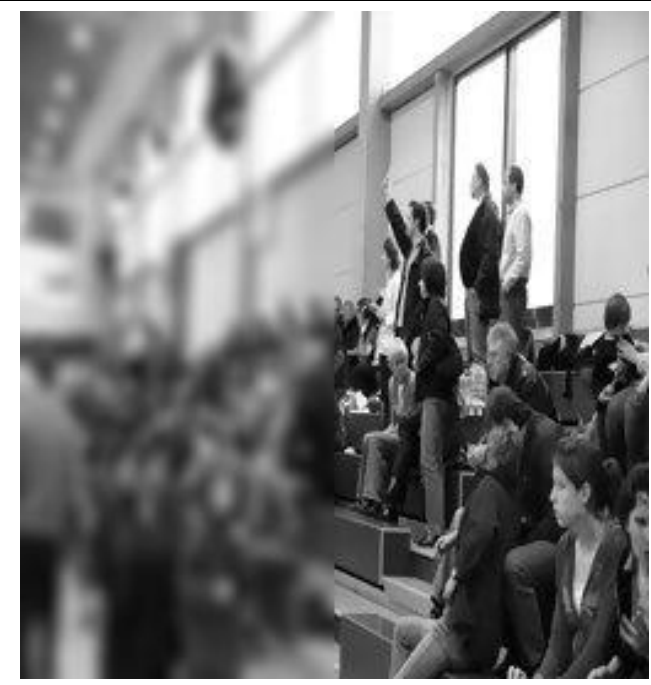

Blurred image1

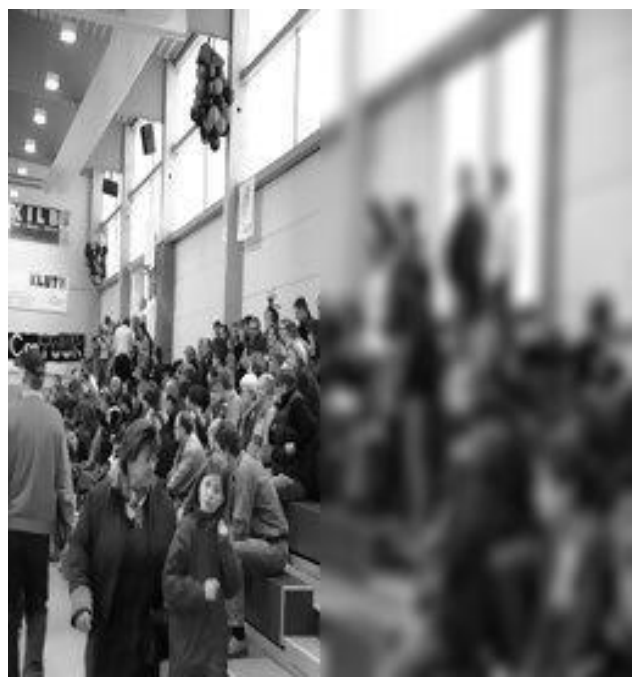

Blurred image2

Figure6. Multi focus images (people)

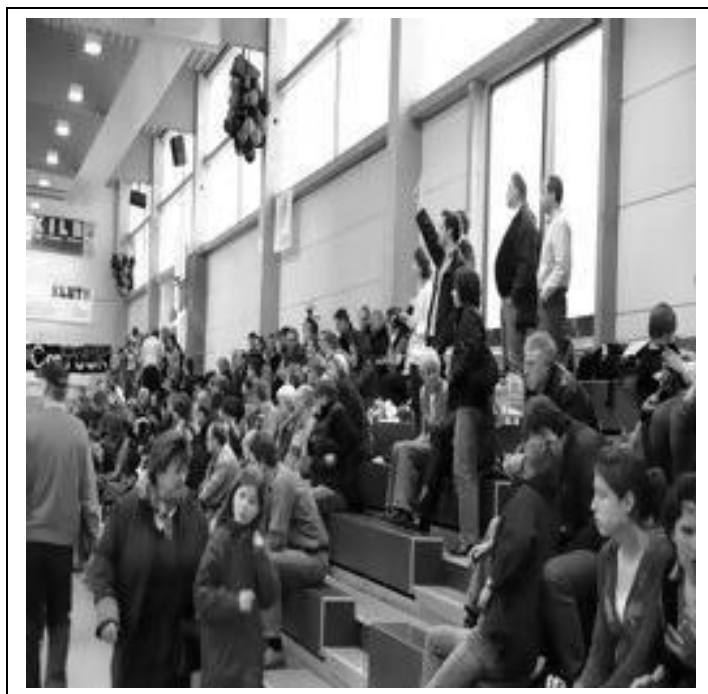

Fusion, proposed method

Figure7. Fused image by proposed method (people) 


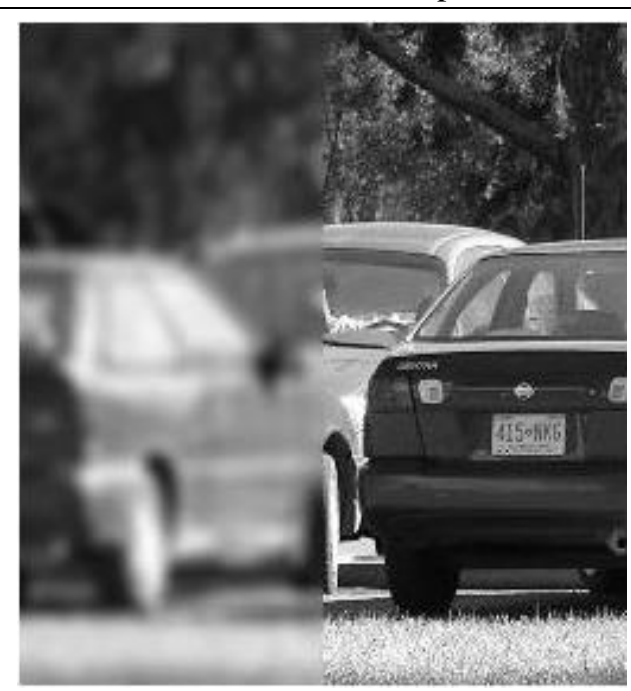

Blurred image1

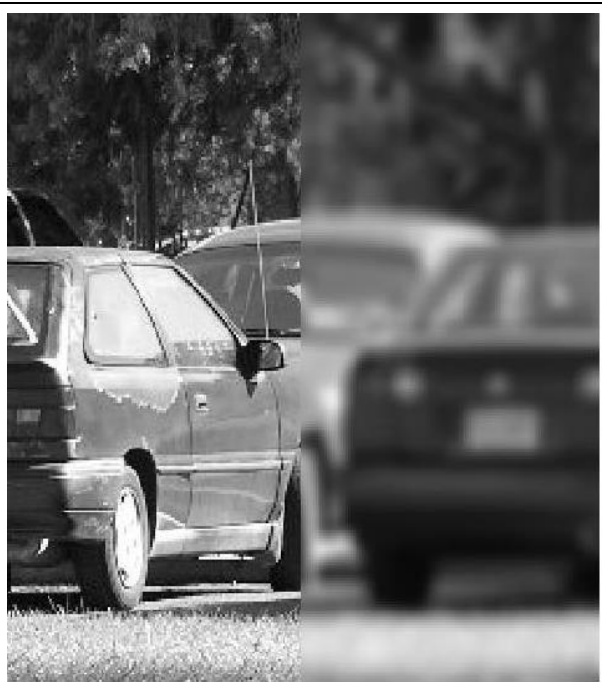

Blurred image2

Figure 8. Multi focus images (cars)

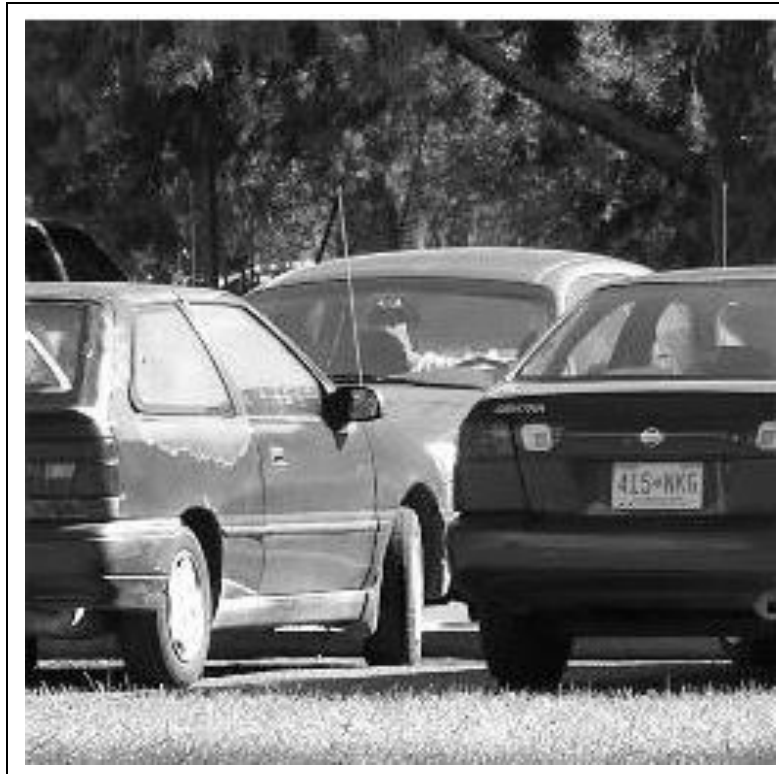

Image fused by proposed method

Figure 9. Fused image by proposed method (cars) 


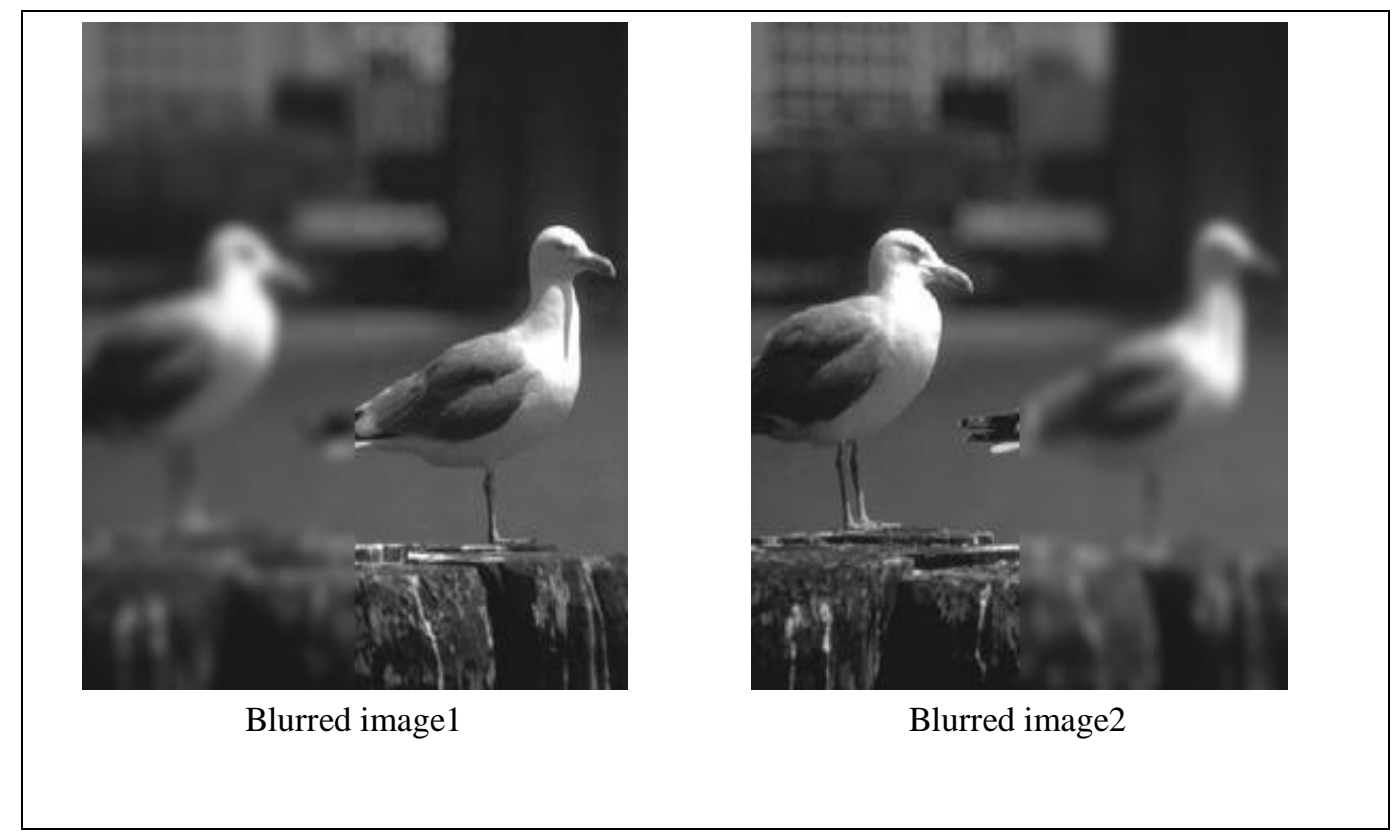

Figure 10. Multi focus images (birds)

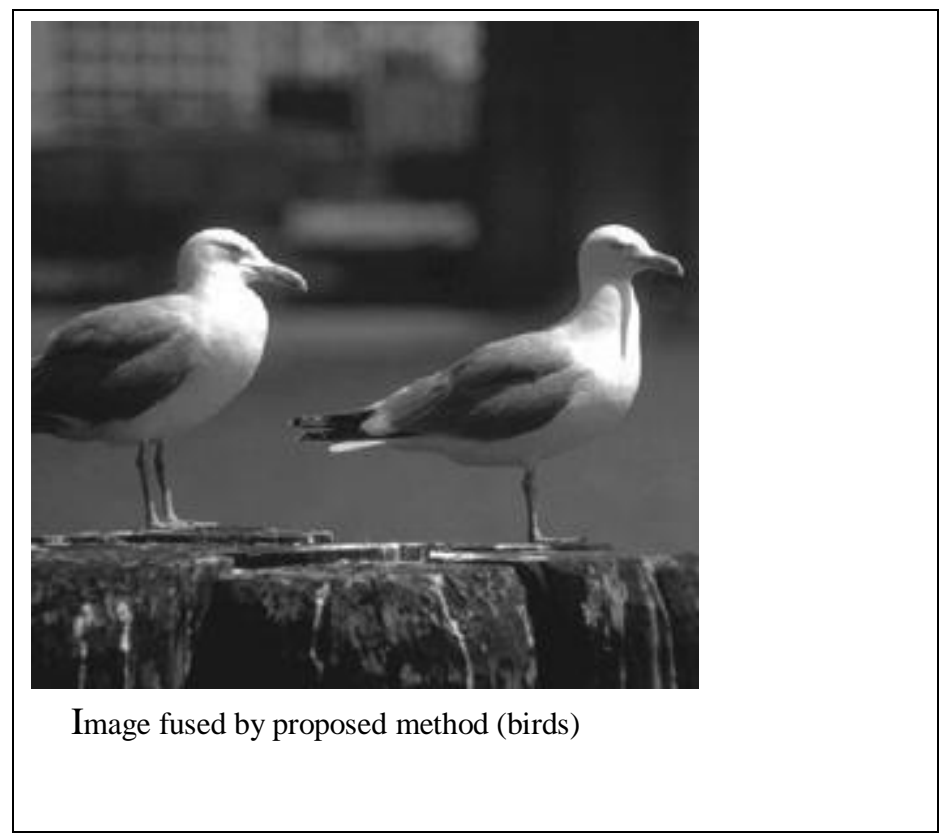

Figure 11. Fused image by proposed method (birds) 


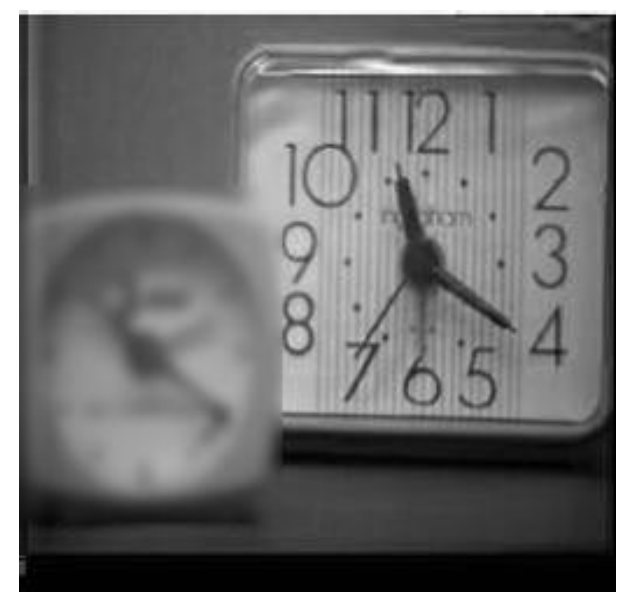

Blurred image1

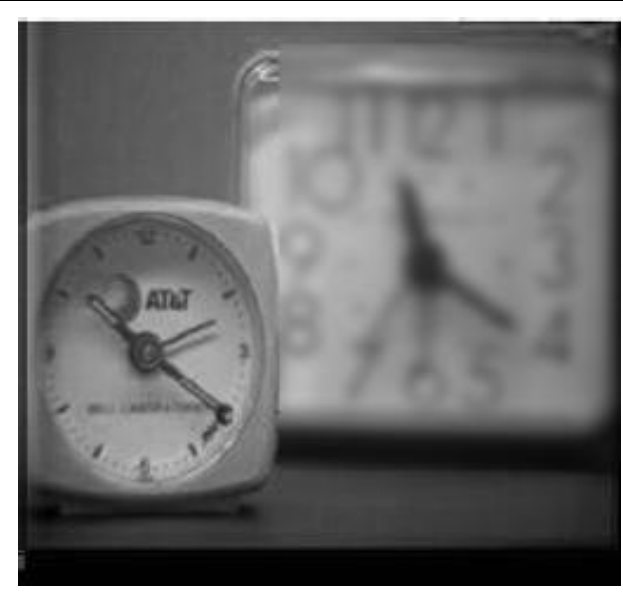

Blurred image2

Figure 12. Multi focus images (clocks)

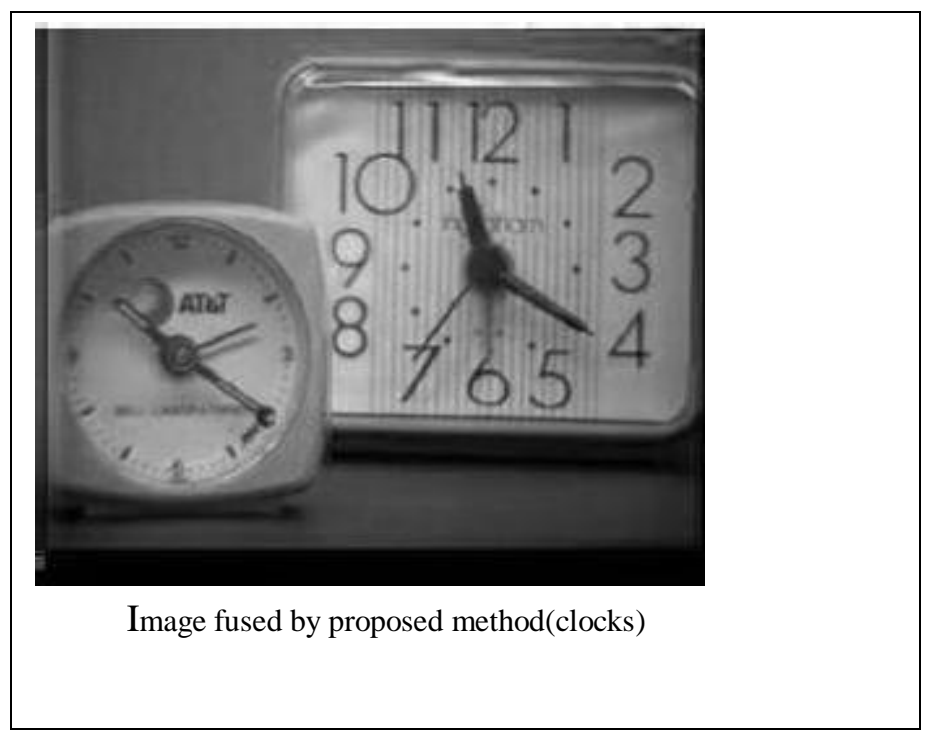

Figure 13. Fused image by proposed method (clocks)

We compared the proposed method to other methods like: Principal Component Analysis (PCA) [5], Decomposition Wavelet Transform (DWT) [8]-[9]and [14]-[15], Bilateral Gradient-based (BG) [21], Laplacian Pyramid with Average as selection rule (LP_AV), Laplacian Pyramid with PCA as selection rule (LP_PCA) [22].

We analyzed the performance of the results using quantitative analysis root mean square error (RMSE) which showed how the pixel values of fused image deviated from the reference image. Let $F(i, j)$ be the gray level intensity of pixel $(i, j)$ of the fused image and $R(i, j)$ be the gray level intensity of pixel $(i, j)$ of the reference image. RMSE between the reference image and fused image wascomputed as: 


$$
R M S E=\sqrt{\frac{1}{m n} \sum_{i=1}^{m} \sum_{j=1}^{n}[R(i, j)-F(i, j)]^{2}}
$$

where $m \times n$ is the size of the input image and $i, j$ represents to the pixel locations. A smaller value of RMSE showed good fusion result. If the value of RMSE was zero then it meant the fused image wasexactly the same as the reference image.

The table1 gives the mean and standard deviation of RMSE for the studded methods.

Table 1. Statistic parameters of the sample (150 images)

\begin{tabular}{|l|l|l|l|l|l|l|}
\hline \multicolumn{1}{|c|}{ Method } & LP_AV & PCA & BG & LP_PCA & DWT & Proposed_method \\
\hline Mean & 8,7452 & 8,7143 & 11,0449 & 8,6043 & 4,1941 & 2,1589 \\
\hline $\begin{array}{l}\text { Standard } \\
\text { deviation }\end{array}$ & 3,8810 & 3,8672 & 4,8593 & 3,8549 & 1,3813 & 0,9437 \\
\hline
\end{tabular}

The results showed that the proposed method has a smaller mean of the RMSE. The histograms of RMSE for 150 images by different methods (Figure 14, 15, 16,17,18 and 19) showed that for all methods the values of RMSE werealmost symmetrically centered around the mean value.

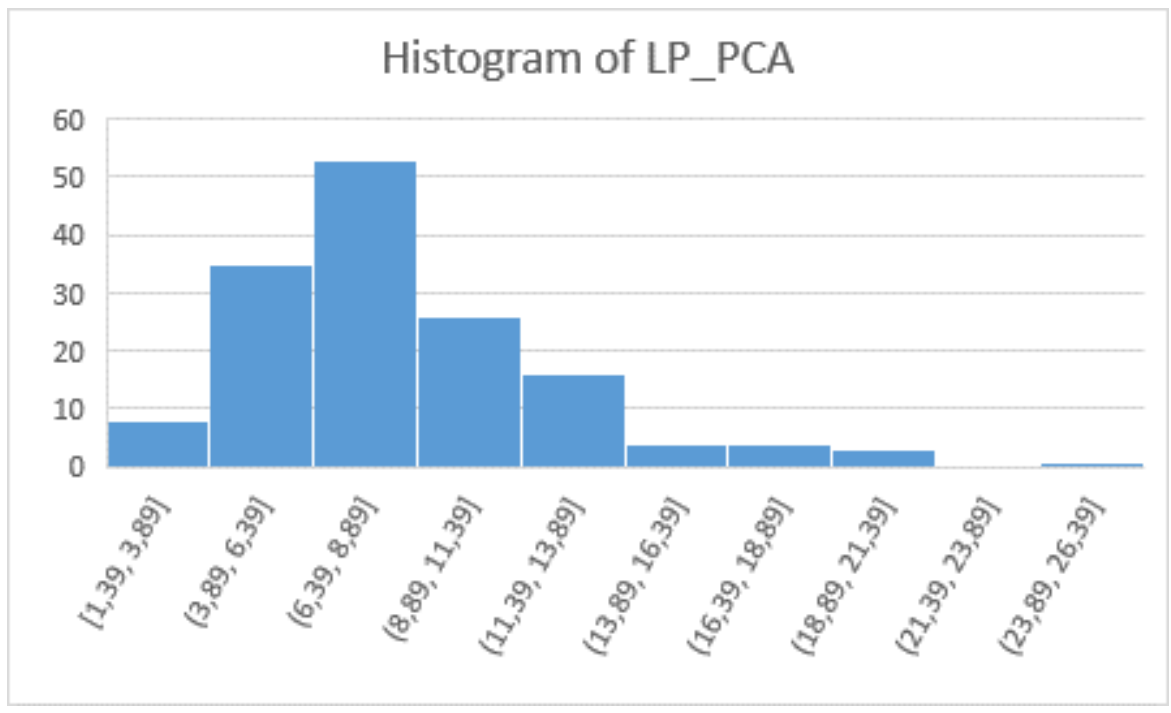

Figure 14. The histogram of LP_PCA method

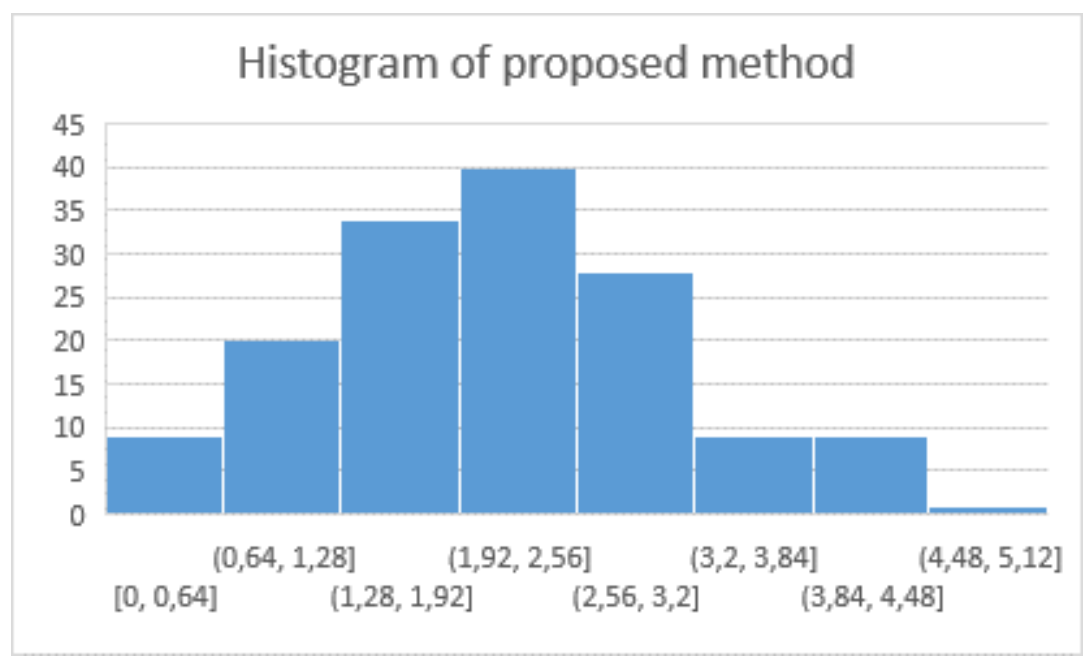


Computer Science \& Information Technology (CS \& IT)

Figure 15. The histogram of proposed method

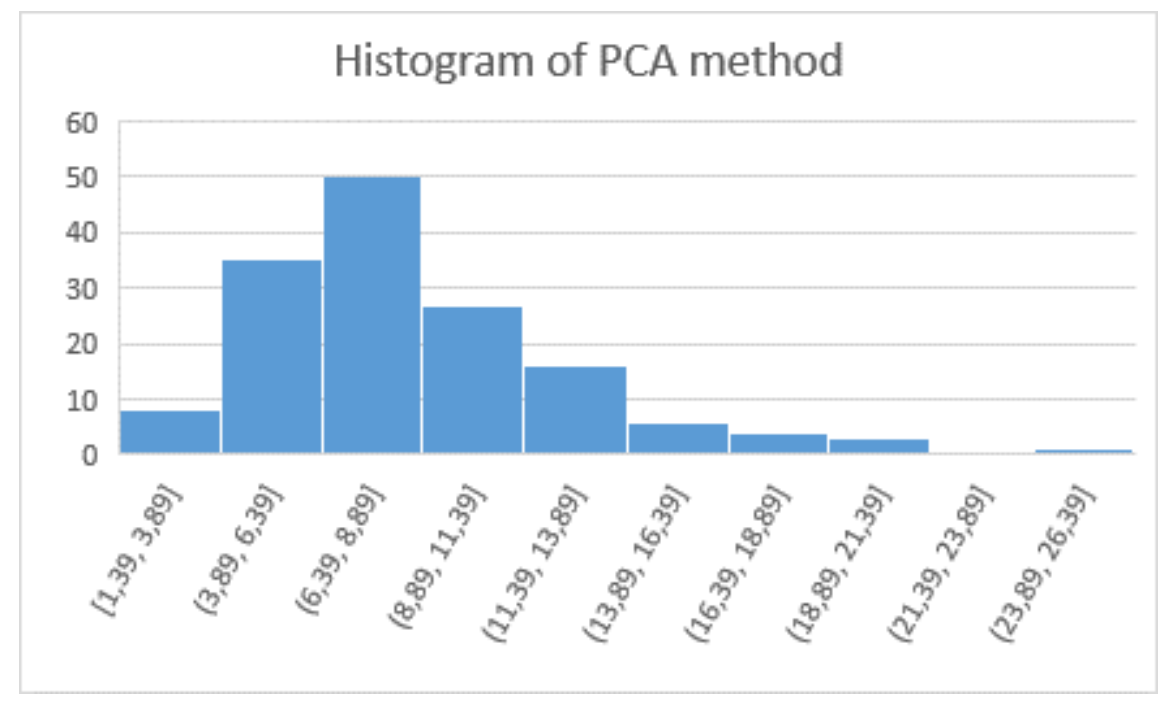

Figure 16. The histogram of PCA method

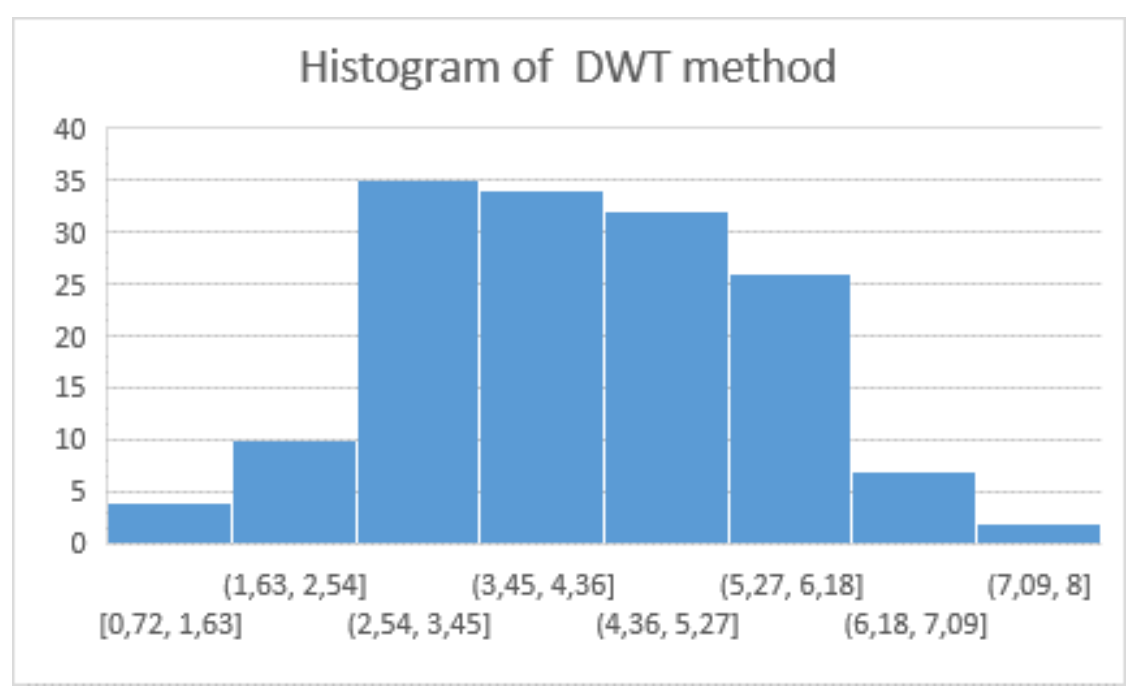

Figure 17. The histogram of DWT method 


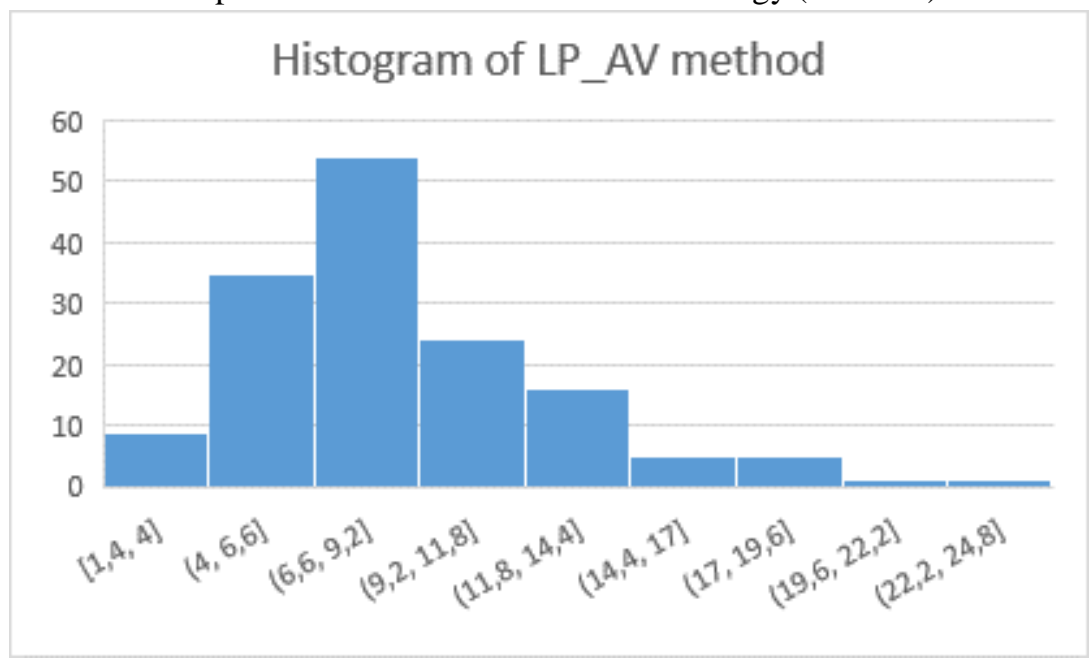

Figure 18. The histogram of LP_AV method

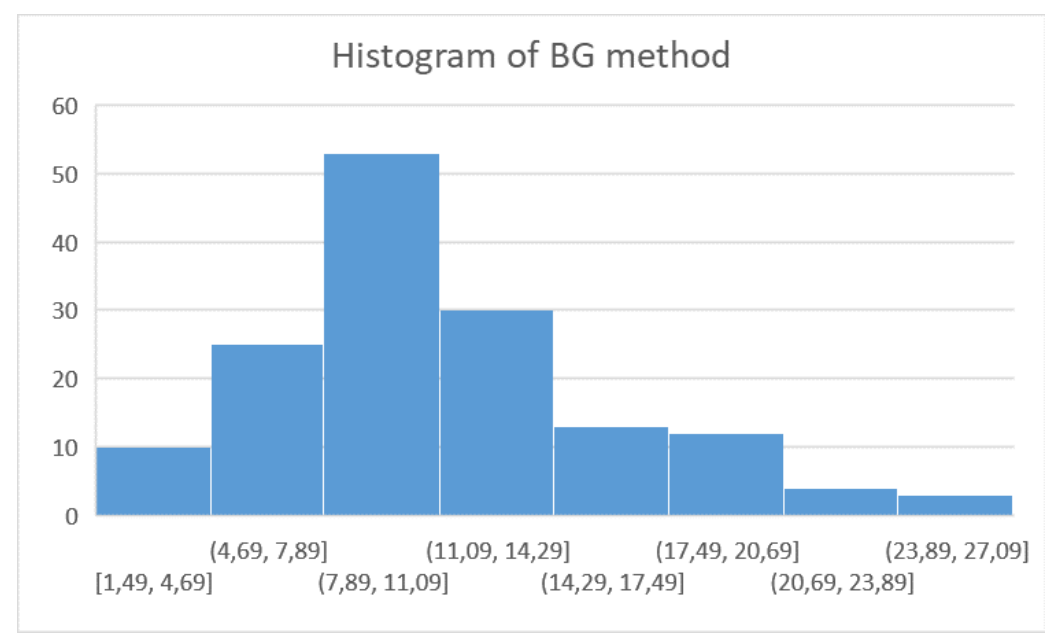

Figure 19. The histogram of LP_PCA method

To compare analyticallythe proposed method to others methods we used the Analysis of variance (ANOVA) with dependent samples (dependence by image). ThesoftwareR gave the following Anova table:

$\begin{array}{lrrrrr} & \text { Df } & \text { Sum Sq } \text { Mean Sq } & \text { F value } & \text { Pr }(>F) \\ \text { Method } & 5 & 8381 & 1676.2 & 473<2 e-16 \\ \text { Residuals } & 745 & 2640 & 3.5 & & \end{array}$

As $\operatorname{Pr}(>\mathrm{F})$ wassmaller than $1 \%$, the methods were significantly different. We used the Newman Keuls test to compare the methods in pairs and make groups having significantly the same mean. The software $\mathrm{R}$ gave the results below of the test. 


$\begin{array}{lrr}\text { \$means } & \text { RMSE } \\ \text { BG } & 11.044867 & 4.859308 \\ \text { DWT } & 4.194067 & 1.381297 \\ \text { LP_AV } & 8.745200 & 3.881025 \\ \text { LP_PCA } & 8.604267 & 3.854875 \\ \text { PCA } & 8.714333 & 3.867235 \\ \text { proposed_M } & 2.158927 & 0.943734 \\ & & \\ \text { \$Comparison } & & \\ \text { NULL } & & \\ & & \\ \text { \$groups } & & \\ & & \\ \text { BG } & 11.044867 & 9 \text { roups } \\ \text { LP_AV } & 8.745200 & \text { b } \\ \text { PCA } & 8.714333 & \text { b } \\ \text { LP_PCA } & 8.604267 & \text { b } \\ \text { DWT } & 4.194067 & \text { C } \\ \text { Droposed M } & 2.158927 & \text { d }\end{array}$

Four different groups: Group "a" contains only method BG having the bigger mean of RMSE (11.045). Group " $b$ " contains 3 methods LP_AV, PCA and LP_PCAthat have significantly the same mean. Group "c" contains only the method DWT which better than group "a" and "b". The last group "d" containing the proposed method that the best method because its mean is the smallest by comparing with other means.

\section{CONCLUSION}

Mutifocus image fusion using Laplacian Pyramid fusion method based on Alpha Stable filter with neighbor alpha stable distance as selection rulegave better result than other studded methods. This method has the advantage of using the local fusionwhile taking into account the neighborhood values.The same technique can be used as a distance to improve Dempster Sheffer method. This work can be havemany applications, such as:

1. Drone is a new technology in digital imaging, it has opened up unlimited possibilities for enhancing photography. Drone can capture images on the same scene that zooms in on different objects, and at various altitudes. It will produces several images on the same scene but with different objects in-focus.

2. For quality control in food industry, cameras are used to take images. Each camera targets one of several objects to detect an anomaly. The objects are on a treadmill. To have animage containing all the objects in clear we can use the proposed methodwhich would give more details

\section{REFERENCES}

[1] Jinjiang Li,1,2 Genji Yuan,1,2 and Hui Fan, "Multifocus Image Fusion Using Wavelet-Domain-Based Deep CNN", Computational Intelligence and Neuroscience, Vol. 2019, 23 pages, 2019.

[2] K. Xu, Z. Qin, G. Wang, H. Zhang, K. Huang, and S. Ye, "Multi-focus image fusion using fully convolutional two-stream network for visual sensors," KSII Transactions on Internet and Information Systems, Vol. 12, no. 5, pp. 2253-2272, 2018 
[3] H. Li, X. He, D. Tao, Y. Tang, and R. Wang, "Joint medical image fusion, denoising and enhancement via discriminative low-rank sparse dictionaries learning," Pattern Recognition, Vol. 79, pp. 130-146, 2018

[4] P. J. Burt and R. J. Kolezynski, "Enhanced image capture through fusion,” in Proc. International Conference on Computer Vision,pp. 173-182, 1993.

[5] W. Wang and F. Chang, "A multi-focus image fusion method based on Laplacian pyramid", Journal of Computers [Online].6(12).Available: http://ojs.academypublisher.com/index.php/jcp/article/view/jcp061225592566, Dec. 2011.

[6] P. Zhao, G. Liu, C. Hu, and H. Huang, "Medical image fusion algorithm on the Laplace-PCA," in Proc. Chinese Intelligent Automation Conference, 2013, pp. 787-794, 2013.

[7] R. Sabre, I Wahyuni “Alpha Stable Filter and Distance for Multifocus Image Fusion”, International Journal of Signal Processing Systems, Vol. 7, No. 2, 2019

[8] G. Pajares and J. M. Cruz, "A wavelet-based image fusion tutorial,” Pattern Recognition, Vol. 37, pp.1855-1872, 2004.

[9] Q. Guihong, Z. Dali, and Y. Pingfan, "Medical image fusion by wavelet transform modulus maxima". Opt. Express [Online].9(4).pp.184-190.Available: http://www.opticsinfobase.org/oe/abstract.cfm?uri=oe-9-4-184, 2001.

[10] H. Li, S. Wei, and Y. Chai, "Multifocus image fusion scheme based on feature contrast in the lifting stationary wavelet domain," EURASIP Journal on Advances in Signal Processing, 2012.

[11] Y. Yang, D. Park, S. Huang, and N. Rao, "Medical image fusion via an effective wavelet-based approach,” EURASIP Journal an Advances in Signal Processing, 2010.

[12] www.rawsamples.ch. Accessed: 15 November 2017.

[13] T. Wan, N. Canagarajah, and A. Achim, "A Statistical Multiscale Image Segmentation via AlphaStable Modelling,” IEEE International Conference on Image Processing, Vol. 4, pp. 357-360, 2017.

[14] Q. Guihong, Z. Dali, and Y. Pingfan, "Medical image fusion by wavelet transform modulus maxima", [Online]. Opt. Express 9(4). pp. 184-190. Available: http://www.opticsinfobase.org/oe/abstract.cfm?uri=oe-9-4-184, 2001.

[15] F. Maes. "Multimodality image registration by maximization of mutual information", IEEE Transaction Medical Imaging. Vol. 16 Issue 2,1997.

[16] P. J. Burt and E. H. Adelson, "The Laplacian pyramid as a compact image code," IEEE Transactions on Communication, Vol. 31, No. 40, Apr. 1983.

[17] P. J. Burt, "The pyramid as a structure for efficient computation,” in Multiresolution Image Processing and Analysis, A. Rosenfeld, Ed., New York: Springer-Verlag, 1984.

[18] I. Wahyuni , "Multi-focus image fusion using local variability", Thèse de doctorat, Université de Bourgogne juin 2017.

[19] S.K. Nayar, "Shape from Focus System,", IEEE Computer Vision and Pattern Recognition, Proceedings CVPR, 1992.

[20] M. Subbarao, "Efficient Depth Recovery through Inverse Optics," Machine Vision for Inspection and Measurement, edited by H. Freeman, Academic Press, 1989. 
[21] J. Tian, L. Chen, L. Ma, and W. Yu, "Multi-focus image fusionusing a bilateral gradient-based sharpness criterion,” Optic Communications, Vol. 284, pp. 80-87, 2011.

[22] V.P.S. Naidu, and. J.R. Raol, "Pixel-level Image Fusion using Wavelets and Principal component Analysis", Defence Science Journal, Vol. 58, No. 3, pp. 338-352. 2008

\section{AUTHORS}

Rachid Sabre received the PhD degree in statistics from the University of Rouen, Rouen, France, in 1993 and Habilitation (HdR) from the University of Burgundy, Dijon, France, in 2003. He joined Agrosup Dijon, Dijon, France, in 1995, where he is an Associate Professor. From 1998 through 2010, he served as a member of Institut de Mathématiques de Bourgogne, France. He was a member of the Scientific Council AgroSup Dijon from 2009 to 2013. Since 2012, he has been a member of Laboratoire Electronique, Informatique, et Image (Le2i), France. He is author/co-author of numerous papers in scientific and technical journals and conference proceedings. His research interests lie in areas of statistical process and spectral analysis for signal and image processing.

Ias Sri Wahyuni was born in Jakarta, Indonesia, in 1986. She earned the B.Sc. and M.Sc. degrees in mathematics from the University of Indonesia, Depok, Indonesia, in 2008 and 2011, respectively.In 2009, she joined the Department of Informatic System, Gunadarma University, Depok, Indonesia, as a Lecturer. She is currently a PhD student at University of Burgundy, Dijon, France. Her current research interests include statistics and image processing. 